\title{
Lyn modulates Claudin-2 expression and is a therapeutic target for breast cancer liver metastasis
}

\author{
Sébastien Tabariès ${ }^{1,2}$, Matthew G. Annis ${ }^{1,2}$, Brian E. Hsu ${ }^{1,2}$, Christine E. Tam ${ }^{1,2}$, \\ Paul Savage ${ }^{1,2}$, Morag Park ${ }^{1,2,3,4}$, Peter M. Siegel ${ }^{1,2,3}$ \\ ${ }^{1}$ Goodman Cancer Research Centre, McGill University, Montréal, Québec, Canada, H3A 1A3 \\ ${ }^{2}$ Department of Medicine, McGill University, Montréal, Québec, Canada, H3A 1A3 \\ ${ }^{3}$ Department of Biochemistry, McGill University, Montréal, Québec, Canada, H3A 1A3 \\ ${ }^{4}$ Department of Oncology, McGill University, Montréal, Québec, Canada, H3A 1A3 \\ Correspondence to: \\ Peter M. Siegel, e-mail: peter.siegel@mcgill.ca \\ Keywords: breast cancer, liver metastasis, claudins, Src family kinase, Lyn \\ Received: January 26, $2015 \quad$ Accepted: January 31, $2015 \quad$ Published: March 25, 2015
}

\section{ABSTRACT}

Claudin-2 enhances breast cancer liver metastasis and promotes the development of colorectal cancers. The objective of our current study is to define the regulatory mechanisms controlling Claudin-2 expression in breast cancer cells.

We evaluated the effect of several Src Family Kinase (SFK) inhibitors or knockdown of individual SFK members on Claudin-2 expression in breast cancer cells. We also assessed the potential effects of pan-SFK and SFK-selective inhibitors on the formation of breast cancer liver metastases. This study reveals that pan inhibition of SFK signaling pathways significantly elevated Claudin-2 expression levels in breast cancer cells. In addition, our data demonstrate that pan-SFK inhibitors can enhance breast cancer metastasis to the liver. Knockdown of individual SFK members reveals that loss of Yes or Fyn induces Claudin-2 expression; whereas, diminished Lyn levels impairs Claudin-2 expression in breast cancer cells. The Lyn-selective kinase inhibitor, Bafetinib (INNO406), acts to reduce Claudin-2 expression and suppress breast cancer liver metastasis.

Our findings may have major clinical implications and advise against the treatment of breast cancer patients with broad-acting SFK inhibitors and support the use of Lyn-specific inhibitors.

\section{INTRODUCTION}

The liver is a common metastatic site for numerous cancers, with the most prominent source of hepatic metastases originating from colorectal and breast tumors [1]. The formation of liver metastases has a major impact on cancer-related survival due to the vital functions carried out by this organ. Excluding brain metastases, the development of liver metastases is associated with the poorest outcomes relative to loco-regional, bone or lung metastases [2]. Cancer cells that arrive in the liver must contend with unique microenvironmental influences that differ markedly from the primary tumor. These include interactions with specialized cell types within the liver such as sinusoidal endothelial cells, Kupffer cells, Hepatic stellate cells, Pit cells and hepatocytes. Coupled with these new cellular interactions, cancer cells also encounter unique features of the liver architecture that together play significant roles in modulating the ability of cancer cells to seed, colonize and grow in this organ [3]. Together, these parameters greatly influence the selection of cancer cells that are best suited to thrive in the liver.

Recently, components of tight-junctional complexes have emerged as key modulators of the metastatic process $[4,5]$. The main functional components of tight junctions are composed of claudin family members [4], which are also gaining increasing attention as metastatic regulators [6]. In colorectal cancer, Claudin-2 levels are elevated and its expression can be detected in pre-neoplastic conditions, such as inflammatory bowel disease that pre-dispose to colon cancer formation [7, 8]. More recently, Claudin-2 has been identified as an important positive modulator of colon cancer tumorigenicity $[9,10]$. Furthermore, Claudin-2 expression has been reported in fibrolamellar hepatocellular, colorectal and pancreatic adenocarcinomas, as well as in 
liver metastases derived from these cancers [7, 11-13]. In contrast, Claudin-2 expression has been reported to decrease in breast cancers of increasing tumor grade and stage, and low Claudin-2 levels are associated with lymph node metastasis [14, 15]. However, despite reduced expression in primary breast cancers, we have recently demonstrated that Claudin-2 functions as a key mediator of breast cancer metastasis to the liver $[16,17]$.

Our previous studies revealed that Claudin-2 expression was selected for in aggressively liver-metastatic breast cancer cells; whereas, the expression of other cellcell adhesion molecules was decreased [17]. We further demonstrated that Claudin-2 is functionally involved in liver metastasis and highly expressed in liver metastases from breast cancer patients $[16,17]$. The mechanisms through which Claudin-2 enhances breast cancer metastasis to the liver involve enhanced seeding and early-stage survival [16]. Indeed, Claudin-2 enables integrin-dependent tumor cell adhesion to the extracellular matrix components and integrin-independent tumor cell adhesion to hepatocytes $[16,17]$. Finally, Claudin-2 has recently been described as a prognostic biomarker able to predict the likelihood of breast cancer recurrence specifically to the liver [18].

Little is known about the mechanisms that control Claudin-2 expression in solid cancers. The objective of our current study is to define the regulatory mechanisms controlling Claudin-2 expression in breast cancer cells.

\section{RESULTS}

\section{Pan inhibition of c-Src family kinase activity enhances Claudin-2 expression in breast cancer cells}

We previously demonstrated that Claudin-2 expression in murine triple-negative breast cancer cells promotes

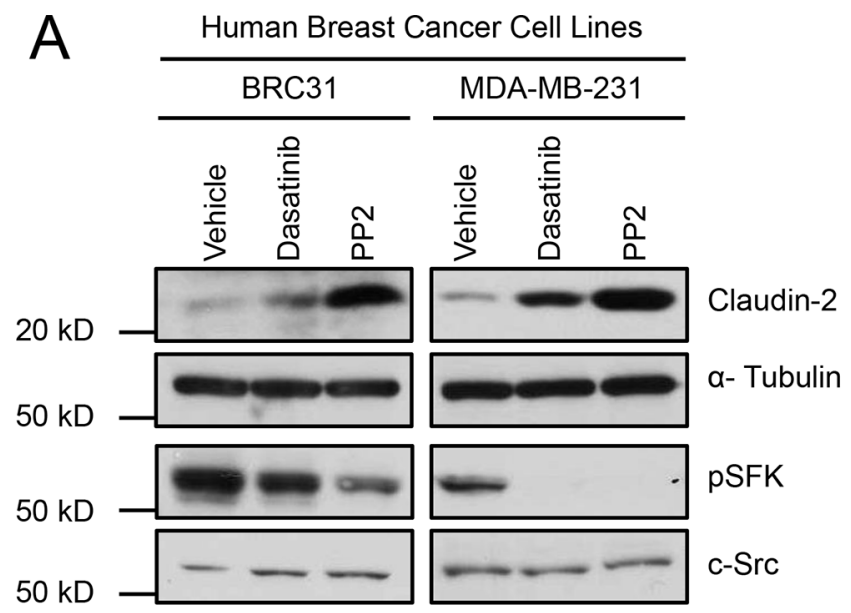

the formation of liver metastases [16, 17]. However, little is known about the mechanisms that govern Claudin-2 expression in breast cancer cells. A previous study has demonstrated that EGFR-dependent activation of the MEK/ERK signaling pathway stimulates Claudin-2 expression in colorectal cancer cells [19].

Signaling via the c-Src Family of non-receptor Kinases (SFK) has been reported to influence cancer cell morphology, adhesion, migration, invasiveness, proliferation, differentiation and survival. SFKs propagate numerous intracellular signals downstream of growth factor receptors, integrin complexes, steroid hormone receptors, $\mathrm{G}$ protein-coupled receptors and via interactions with components of the cytoskeleton [20,21]. Indeed, activation of Src has been correlated with poor outcomes for patients with diverse types of cancer [22, 23]. Lyn, a SFK member, has recently received attention as an important regulator of signaling in basal/triple negative breast cancers. In this breast cancer subtype, Lyn expression correlated with poor survival and increased likelihood of recurrence [24, 25]. We have employed triple negative breast cancer models, expressing Claudin-2 (MDA-MB-231 and BRC31) [17, $26,27]$, to elucidate the role of Claudin-2 as a promoter of the breast cancer liver metastatic phenotype [16, 17]. Thus, we investigated the involvement of SFKs in the regulation of Claudin-2 expression and liver metastatic ability of breast cancer cells. To accomplish this, we treated breast cancer cells with Dasatinib or PP2, two independent pharmacological pan-SFK inhibitors. We observed an increase in Claudin-2 levels when human (Figure 1A) and mouse (Figure 1B) breast cancer cells were individually treated with each SFK inhibitor.

We next assessed whether pan-SFK inhibitors affected transcription of CLDN2 in breast cancer cells. In agreement with our immunoblotting results, quantitative

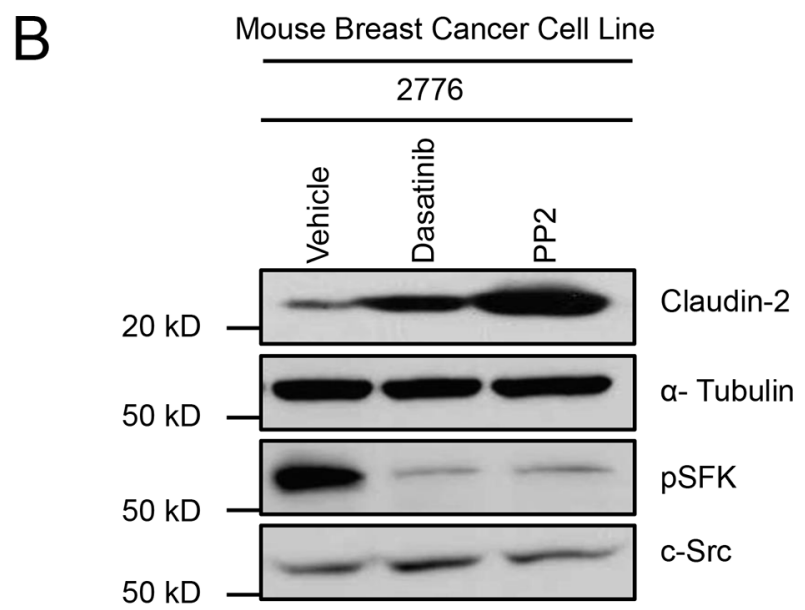

Figure 1: Inhibition of Src family kinases (SFK) enhances Claudin-2 expression in breast cancer cells. Immunoblot analysis for Claudin-2 expression in both human breast (BRC31, MDA-MB-231) (A) or in a liver metastatic variant (2776) derived from the mouse 4T1 breast cancer cell line (B) treated with vehicle or two SFK inhibitors (Dasatinib and PP2). $\alpha$-Tubulin served as a loading control and pSFK/Src blots revealed the efficacy of the SFK kinase inhibitors. 
real-time PCR showed that CLDN2 mRNA levels are increased in both human and mouse breast cancer cells following treatment with pan-SFK inhibitors $(1.73-3.33$ fold induction for Dasatinib; 6.51 - 30.7 fold induction for PP2; Supplementary Figure 1A-1D). These results indicate that an SFK signaling pathway regulates CLDN2 expression at the transcriptional level in breast cancer cells.

The EGFR-MEK-ERK1/2 pathway has been implicated in the transcriptional regulation of CLDN2 in A549 lung adenocarcinoma cells through binding of the transcription factors, c-Fos and c-Jun, to the human CLDN2 promoter region via an AP-1 binding site [28]. Phosphorylation of c-Fos (p-c-Fos) leads to stabilization of this transcription factor and enhanced transcriptional activity of the AP-1 complex [29]. Therefore, we assessed the effect of SFK inhibitors on the levels of p-c-Fos in breast cancer cells. Treatment of MDA-MB-231 breast cancer cells with Dasatinib or PP2 resulted in elevated levels of p-c-Fos (Ser374 and Ser32) and compared to total c-Fos levels, which remained unchanged (Figure 2A). Similar results were obtained using 4T1-derived mouse liver-metastatic breast cancer cells (Figure 2B). Interestingly, we observed a reduction in p-c-Jun (S63) and total c-Jun levels following treatment with pan-SFK inhibitors in both human (Figure 2A) and mouse (Figure 2B) breast cancer cells. These antibodies do not recognize JunB or JunD, raising the possibility that these Jun family members could heterodimerize with c-Fos.

We then used chromatin immunoprecipitation assays to monitor the recruitment of c-Fos to the human CLDN2 promoter in MDA-MB-231 breast cancer cells following treatment with SFK inhibitors. As expected, a significant increase in c-Fos recruitment was observed at the CLDN2 promoter in cells treated with inhibitors compared to controls (Figure 2C). These results demonstrate that SFKs act to suppress recruitment of c-Fos to the AP1 binding site within the human CLDN2 promoter in breast cancer cells, which is relieved upon treatment with SFK inhibitors.

\section{Dasatinib treatment increases the formation of breast cancer liver metastases}

Given our previous data supporting Claudin-2 as an important promoter of breast cancer liver metastasis [16, 17], we assessed the effect of Dasatinib treatment on the formation of liver metastases following intra-splenic injection of 2776 liver-aggressive breast cancer cells that expressed endogenous Claudin-2 levels and 2776 cells that had stably reduced Claudin-2 expression (Figure 3A). We observed that mice treated with Dasatinib exhibited a 2.6-fold increase in the number of liver metastases and a 8.3-fold increase in the liver metastatic burden compared to animals receiving the vehicle control (Figure 3B and 3C). To determine if the increase in the liver metastatic burden was dependent on Claudin-2, we included a cohort of mice that received Dasatinib treatment but were injected with 2776 liver-aggressive cells harboring shRNAs targeting Claudin-2. Immunoblot analysis demonstrated that the presence of the shRNAs targeting Claudin-2 were able to almost completely suppress
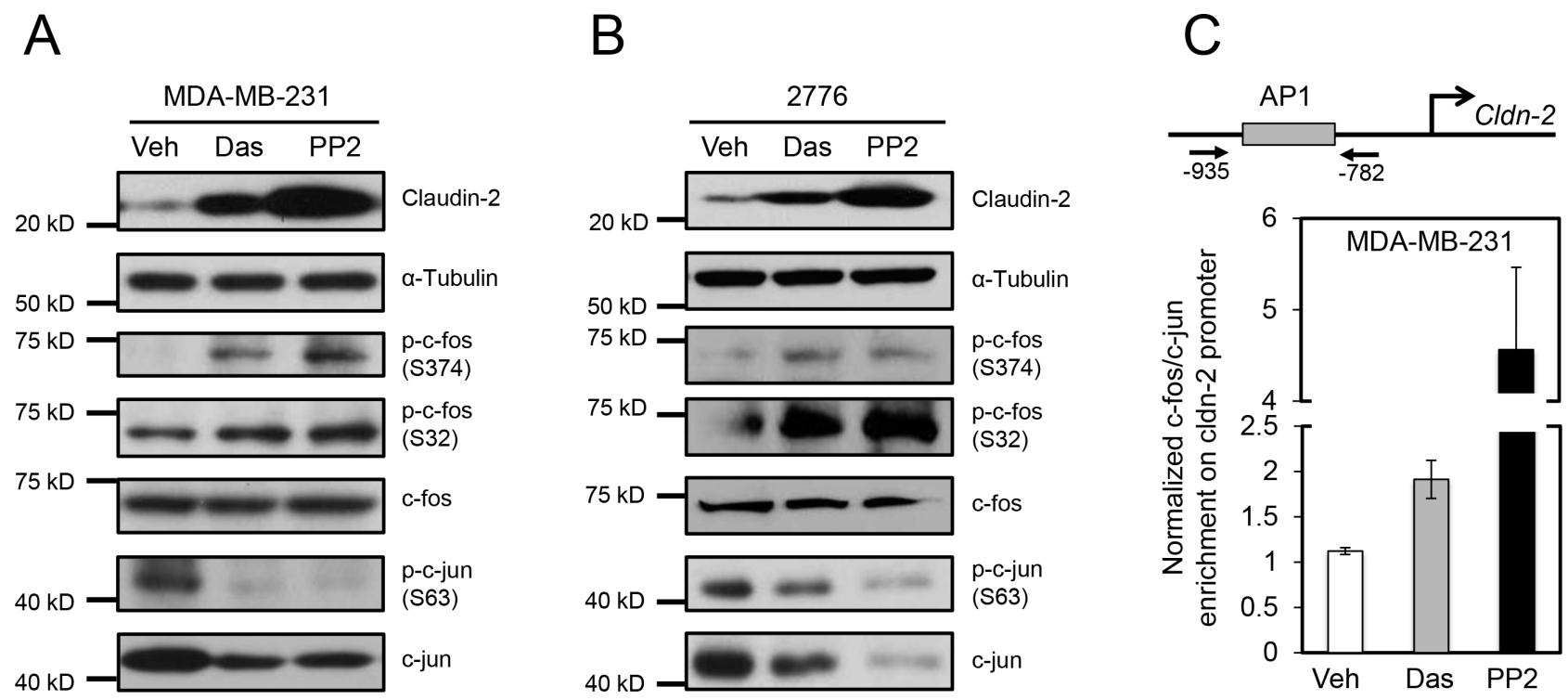

Figure 2: Differential phosphorylation and recruitment of c-Fos containing complexes to the AP1 site of the Claudin-2 promoter are associated with the changes in Claudin-2 expression following treatment with c-Src family kinase (SFK) inhibitors. Treatment of human breast cancer cells (MDA-MB-231) (A) or the liver metastatic variant (2776) derived from the mouse 4T1 breast cancer cell line (B) with SFK inhibitors results in enhanced c-Fos phosphorylation (p-c-Fos) and elevated Claudin-2 expression. Diminished c-Jun phosphorylation (p-c-Jun) and total c-Jun levels are observed following treatment of breast cancer cells with SFK inhibitors. Immunoblots for $\alpha$-Tubulin served as loading controls. (C) Chromatin immunoprecipitation experiments reveal that c-Fos/c-Jun complexes are enriched on the AP1 site within the CLDN2 promoter in MDA-MB-231 breast cancer cells. 


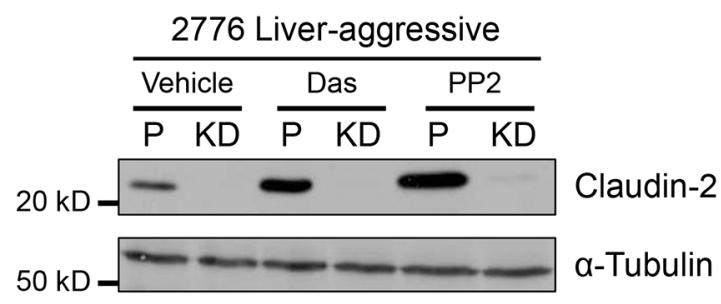

B

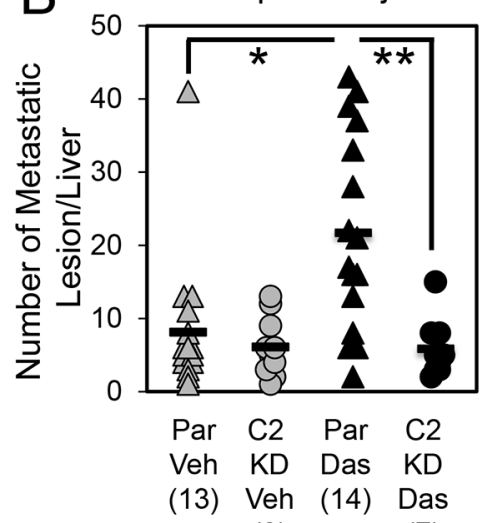

(8)

(7)

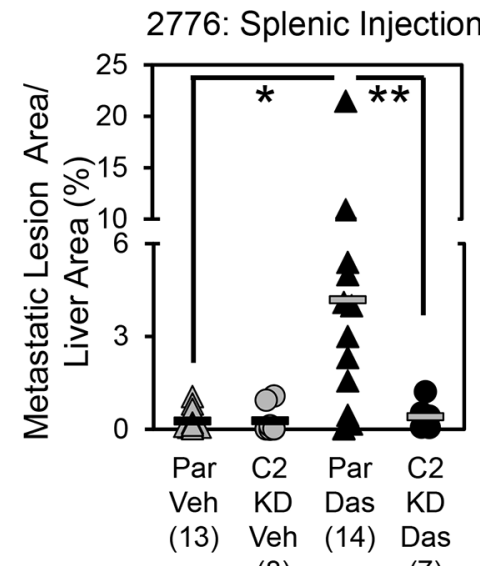

(8)

(7)

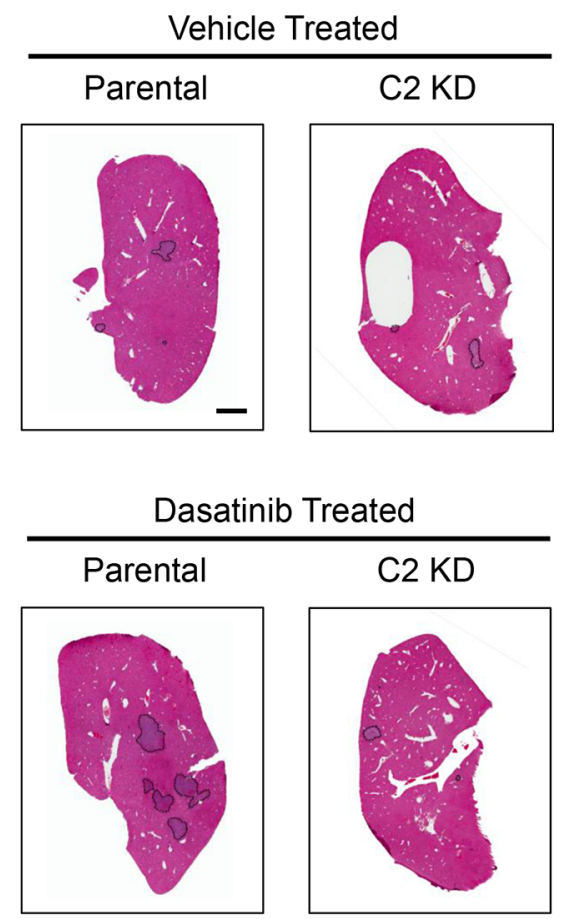

Figure 3: Dasatinib treatment enhances the formation of breast cancer liver metastases. (A) Immunoblot analysis of Claudin-2 expression following treatment with c-Src family kinase inhibitors in parental 2776 cells (P) or 2776 cells harboring shRNAs against endogenous Claudin-2 (KD). As a loading control, total cell lysates were blotted for $\alpha$-Tubulin. (B) Dasatinib treatment increases the number of metastases and the liver-metastatic burden derived from parental 2776 cells, but not from cells with diminished Claudin-2 levels, following splenic injection $\left(1 \times 10^{4}\right.$ cells). A statistically significant increase in both the number of hepatic metastases or the liver metastatic burden is observed when the control cohort (Par Veh) is compared to the Dasatinib-treated cohort (Par Das) $(*, P<0.015)$. In contrast, this effect is lost when a cell population with diminished Claudin-2 expression was injected (C2 KD Das) (**, $P<0.012$, Par Das vs $\mathrm{C} 2 \mathrm{KD}$ Das). The number of mice analyzed in each cohort is indicated in parentheses. (C) Representative images of H\&E stained liver sections exhibiting the liver metastatic burden in each cohort. Scale bar represents $2 \mathrm{~mm}$ and applies to all panels. Par, Parental; Veh, Vehicle; Das, c-Src family kinase inhibitor (Dasatinib); PP2, Src family kinase inhibitor.

the increase in Claudin-2 expression that is normally seen following Dasatinib treatment (Figure 3A). Interestingly, mice injected with 2776 breast cancer cells harboring Claudin-2 targeting shRNAs failed to exhibit an elevated liver metastatic burden in response to Dasatinib treatment (Figure 3B and 3C).

To better understand the increase in the livermetastatic burden following Dasatinib treatment, we examined the proliferative and apoptotic indices in the resulting lesions. We observed no differences in the degree of tumor cell proliferation (Ki67) in liver metastases derived from mice treated with vehicle or Dasatinib (Supplementary Figure 2A). Likewise, no statistically significant differences were observed in the number of apoptotic cells (Cleaved Caspase-3) within the liver metastases derived from these cohorts (Supplementary Figure 2B). However, in agreement with the increase of Claudin-2 expression following Dasatinib treatment that we observe in vitro (Figure 1B), immunohistochemical analysis revealed a clear increase in Claudin-2 positivity within hepatic metastases arising in the Dasatinib-treated cohort (Supplementary Figure 2C). Thus, the Dasatinib-induced increase in Claudin-2 expression may promote breast cancer survival and enhanced formation of liver metastases, as we have described previously [16].

\section{Diminished Lyn expression suppresses Claudin-2 levels while reduction of Fyn or Yes levels enhances Claudin-2 expression in breast cancer cells}

Given the implication of these results for the treatment of breast cancer patients with pan-SFK inhibitors, we next investigated the involvement of individual SFK members (c-Src, Fyn, Yes or Lyn) in the regulation of Claudin-2 expression. To do so, we stably diminished the expression of each SFK in human breast cancer cells (MDA-MB-231 and BRC31) using shRNA-mediated approaches (Figure 4). No major effect on Claudin-2 levels was observed with single knockdown of c-Src (Figure 4A). However, individual loss of either Yes or Fyn, the latter not being expressed by BRC31 cells, resulted in a dramatic increase in Claudin-2 expression (Figure 4B and 4C), which recapitulates the effects seen with pan-SFK inhibitors (Figure 1A). Similar results were observed in the 2776 liver-aggressive cell population 
A

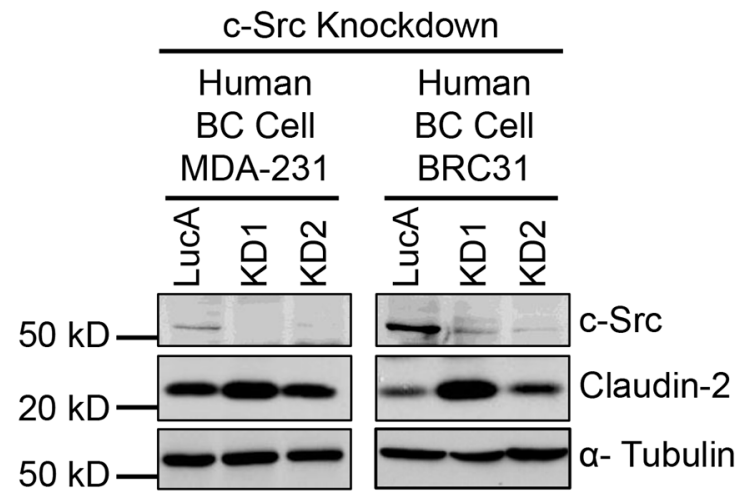

B

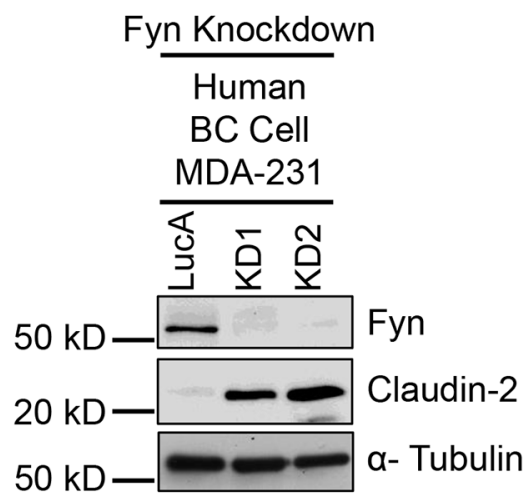

D

Figure 4: Reduction of Fyn or Yes expression increases Claudin-2 levels while reduced Lyn expression lowers Claudin-2 levels in breast cancer cells. Immunoblot analysis of Claudin-2 expression in human breast cancer cells (MDA-MB-231 and BRC31) harbouring a control vector (LucA) or infected with two independent shRNA expression vectors (KD1 and KD2) against c-Src (A), Fyn (B), Yes (C) or Lyn (D). As a loading control, total cell lysates were blotted for $\alpha$-Tubulin.

(Supplementary Figure 3A and 3B). Intriguingly, decreased Lyn levels significantly reduced Claudin-2 expression in human (Figure 4D) and mouse triple-negative breast cancer cells (Supplementary Figure 3C). We also observed that individual loss of Yes expression, and to a lesser extent Fyn, resulted in elevated Lyn levels in both MDA-MB-231 and BRC 31 breast cancer cells (Supplementary Figure 4A and $4 \mathrm{~B}$ ). Taken together our observations reveal a critical role for Lyn in promoting Claudin-2 expression in breast cancer cells, highlighting the potential of this specific SFK family member as an important therapeutic target in the management of liver metastasis.

\section{Dasatinib (pan-SFK inhibitor) treatment enhances, while Bafetinib (Lyn-selective inhibitor) suppresses, Claudin-2 expression in breast cancer patient-derived xenograft explants}

We next examined the potential relevance of our findings to additional human breast cancer models using a breast cancer patient-derived xenograft (PDX) developed in-house (GCRC1735) or a previously described triple negative breast cancer patient-derived xenograft (HCI010) [30]. As observed with human (MDA-MB-231 and BRC31) and mouse (2776) breast cancer cells (Figure 1), we observed an increase in Claudin-2 levels when GCRC1735 PDX cells were treated with Dasatinib (Figure 5A). However, no change in Claudin-2 levels was observed in the HCI010 PDX explant (Figure 5B).

Given the central role Lyn plays in regulating Claudin-2 expression, kinase inhibitors that specifically target Lyn could potentially reduce Claudin-2 expression and breast cancer liver metastasis. Bafetinib is a kinase inhibitor that is highly selective for Lyn and BCR/ABL [31-33] and exhibits little activity against other members of the c-Src Family, with the exception of Lck [34]. Interestingly, Bafetinib treatment dramatically reduced Claudin-2 levels in both the GCRG1735 or HCI010 PDX cultures. These data are in agreement with our results using shRNA-mediated knockdown of Lyn, which resulted in 


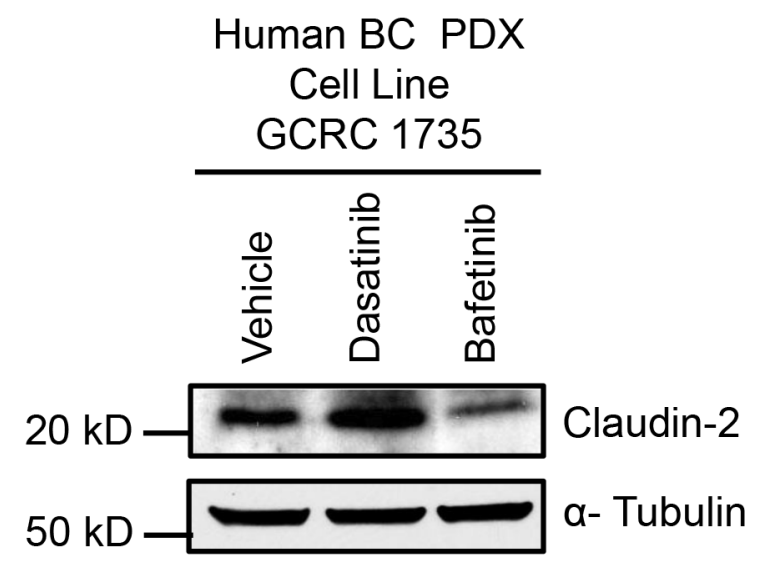

B

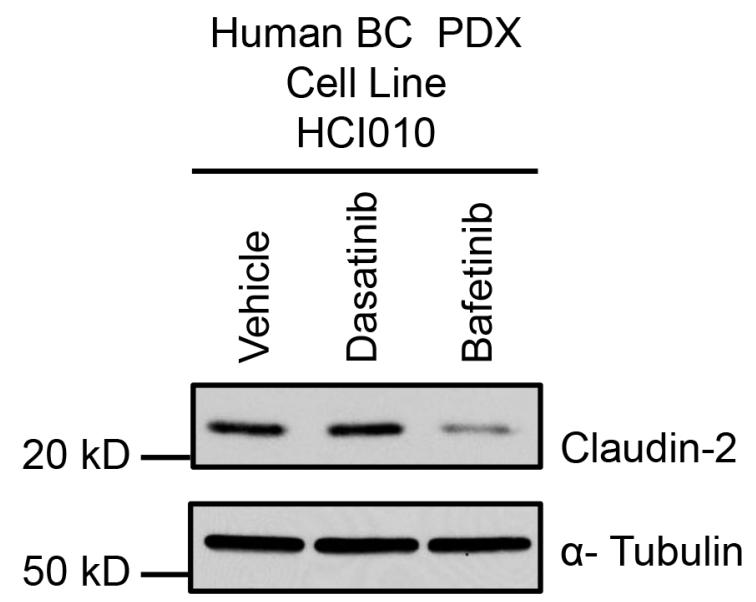

Figure 5: Dasatinib treatment increases, while Bafetinib treatment lowers, Claudin-2 levels in breast cancer patient derived xenograft explants. Immunoblot analysis of Claudin-2 expression in GCRC1735 (A) or HCI010 (B) explants derived from triple negative breast cancer human patient derived following treatment with either Dasatinib $(30 \mathrm{nM})$ or Bafetinib $(10 \mu \mathrm{M})$ inhibitor. As a loading control, total cell lysates were blotted for $\alpha$-Tubulin.

diminished Claudin-2 expression in both human and mouse breast cancer cells (Figure 4D; Supplementary Figure 3C).

\section{Bafetinib decreases the formation of breast cancer liver metastases}

We also assessed whether Bafetinib treatment had the same effect on Claudin-2 levels in multiple breast cancer cell models. Uniformly, Bafetinib treatment reduced Claudin-2 levels in human breast cancer cells and liver-metastatic mouse breast cancer cells (Figure 6A and 6B). We next determined whether Bafetinib treatment would act to suppress the formation of breast cancer liver metastasis in vivo. Following intra-splenic injection of
2776 liver-aggressive breast cancer cells, we observed that mice treated with Bafetinib exhibited a 4-fold decrease in number of hepatic lesions and a 2.3-fold decrease in the formation of liver metastases compared to animals receiving the vehicle control (Figure $6 \mathrm{C}$ and $6 \mathrm{D}$ ). We observed a slight decrease in tumor cell proliferation (Ki67) in hepatic metastases derived from 2776 liver-aggressive breast cancer cells that emerged in mice treatment with Bafetinib (Supplementary Figure 5A). In contrast, the degree of apoptosis (Cleaved Caspase-3) was unchanged in liver metastases that grew in the vehicle or Bafetinibtreated cohorts (Supplementary Figure 5B). Interestingly, the levels of Claudin-2 were clearly diminished in liver metastases from mice treated with Bafetinib compared 
A

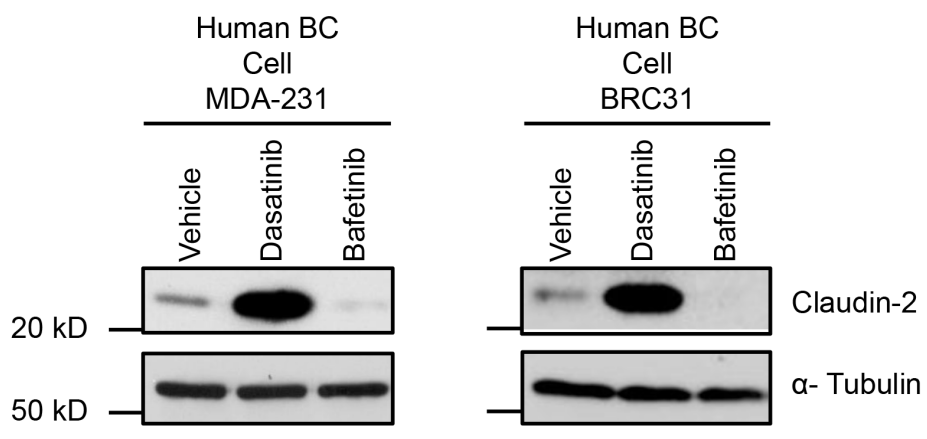

C

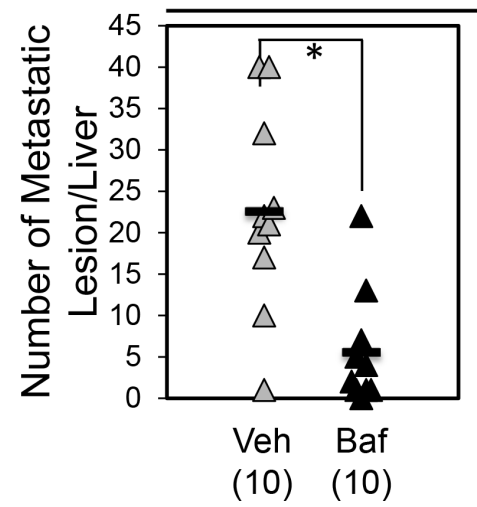

2776: Splenic Injection

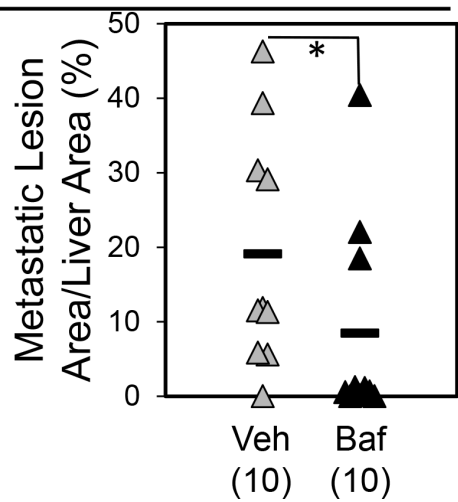

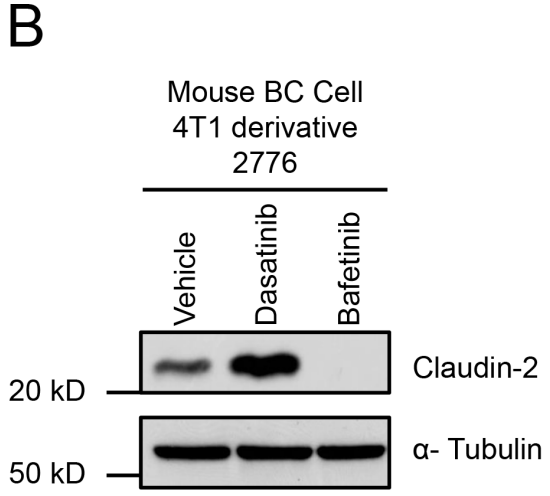

D 2776: Splenic Injection

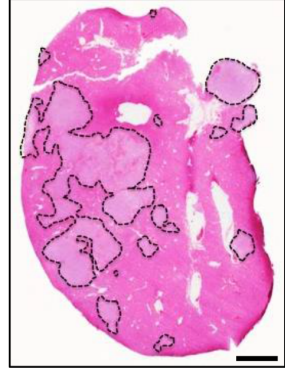

Vehicle Treated

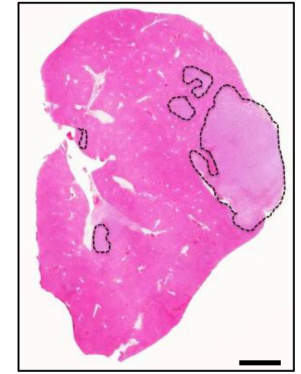

Bafetinib Treated

Figure 6: Bafetinib treatment impairs the formation of breast cancer liver metastases. Immunoblot analysis of Claudin-2 expression in both human breast cancer cells (MDA-MB-231 and BRC31) (A) or 2776 mouse breast cancer cells (B) following treatment with Bafetinib inhibitor. As a loading control, total cell lysates were blotted for $\alpha$-Tubulin (A, B). (C) Bafetinib treatment decreases the formation of liver metastases derived from 2776 cells following splenic injection $\left(1 \times 10^{5}\right.$ cells $)$. A statistically significant decrease in both the number of hepatic metastatic lesions or the liver metastatic burden is observed when the control cohort (Par Vehicle) is compared to the Bafetinib-treated cohort (Par Baf) $(*, P<0.05)$. The number of mice analyzed in each cohort is indicated in parentheses. (D) Representative images of H\&E stained liver sections exhibiting the liver metastatic burden in each cohort. Scale bar represents $2 \mathrm{~mm}$. Veh, Vehicle; Baf, Lyn inhibitor (Bafetinib).

to vehicle-treated controls (Supplementary Figure 5C), which recapitulate our in vitro findings (Figure $6 \mathrm{~A}$ and 6B). Together, these observations suggest that, in contrast with the use of Dasatinib (Figure 3), breast cancer patients may benefit from treatment with Bafetinib to manage the formation of liver metastases.

Our previous experiments were conducted with one liver-aggressive cell population (2776) and the Dasatinib and Bafetinib experiments were conducted in separate cohorts. To confirm these observations, we assessed the effect of Dasatinib or Bafetinib treatment on an independent liver-aggressive breast cancer population (2792). As expected, we observed an increase in Claudin-2 levels when cells were treated with Dasatinib and a decrease in Claudin-2 levels in Bafetinib-treated cells (Supplementary Figure 6A). Following intra-splenic injection, mice treated with Dasatinib exhibited a 2.4-fold increase in the number of liver metastasis and a 3.3-fold increase in the liver metastatic burden compared to animals receiving the vehicle control (Supplementary Figure 6B-6D). In contrast, mice treated with Bafetinib exhibited a 2.1-fold decrease in the number of liver metastasis and a 3.6-fold decrease in the liver metastatic burden compared to the cohort that received vehicle alone (Supplementary Figure 6B-6D). Together, those observations suggest that treating breast cancer patients with Dasatinib (pan-SFK) could have the unanticipated consequence of promoting breast cancer liver metastasis formation whereas breast cancer patients may benefit from Bafetinib (Lyn-selective) treatment.

\section{DISCUSSION}

Our previous studies demonstrated that Claudin-2 is functionally involved in breast cancer metastasis to the liver $[16,17]$ and a recent independent study has highlighted the potential of Claudin-2 as a prognostic biomarker that is able to predict the liver metastatic potential of primary breast tumors [18]. Our current findings reveal, for the first time, that signaling via Src family kinases can control Claudin-2 expression in breast cancer cells. 
Using reporter assays and chromatin immunoprecipitation approaches, it has recently been shown that the EGFR-MEK-ERK1/2 pathway can regulate the transcription of CLDN2 in A549 lung adenocarcinoma cells [28]. In these cells, activation of the EGFR pathway led to enhanced binding of the transcription factors, c-Fos and c-Jun, to the human CLDN2 promoter region via an AP-1 binding site [28]. Intriguingly, increased AP-1 activity, induced by ectopic overexpression of c-Jun in SKBR3 breast cancer cells, resulted in a significant increase in liver metastasis following tail vein injection [35]. Our current results are in agreement with this mechanism as the application of pan-SFK pharmacological inhibitors increased the transcriptional activity of the AP- 1 complex, as measured by the phosphorylation status of c-Fos (Figure 2A and 2B) and its recruitment to the AP-1 binding site of the human $C L D N 2$ promoter in breast cancer cells (Figure $2 \mathrm{C}$ ). In contrast to lung adenocarcinoma cells or HER2+ breast cancer cells, we observed no evidence for a potential partnership role for c-Jun as the total levels of c-Jun and its phosphorylation (S63) were diminished in Dasatinib treated cells. Thus, it is conceivable that CLDN2 expression in triple-negative breast cancer cells is controlled by an AP-1 complex composed of c-Fos and another Jun family member, such as Jun B or JunD. These data demonstrate that the transcription of Claudin-2 can be regulated through an SFK-c-Fos pathway.

Given the role of this SFK-c-Fos pathway in regulating Claudin-2 expression, we predicted that panSFK inhibitors would, in fact, enhance breast cancer metastasis to the liver, through the transcriptional upregulation of Claudin-2. We demonstrate that Dasatinib administration promotes the liver metastatic ability of breast cancer cells in pre-clinical mouse models (Figure 3 and Supplementary Figure 6). The Dasatinib-induced increase in liver metastasis is mediated by Claudin-2 as knockdown of Claudin-2 completely abolishes the Dasatinib-mediated effect. We did not observe significant differences in cellular proliferation and apoptosis in liver metastases treated with Dasatinib, thus, it is likely that Dasatinib-induced Claudin-2 expression may enhance the early colonization steps of the liver-metastatic breast cancer cells as we have previously described [16].

These results are concerning given that SFK inhibitors have been employed as therapeutic agents in the setting of triple-negative metastatic breast cancer. A recent phase II study failed to demonstrate significant promising results that would justify the use of Saracatinib as a monotherapy in hormone receptor negative metastatic breast cancer [36]. In contrast, an independent phase II study has reported a modest clinical benefit with Dasatinib, which resulted in a partial response (2 patients) or stable disease (11 patients) in 43 evaluable patients with advanced triple-negative breast cancer [37]. Similarly, a phase II clinical study conducted in 70 patients with HER2-amplified tumors or estrogen receptor (ER) and/or progesterone receptor (PR)-positive tumors revealed that nine patients with ER+ and/or PR+ tumors had a partial response or stable disease when treated with Dasatinib [38]. Taken together, the results of several Phase II studies a revealed that Src inhibitors have shown little efficacy as a monotherapy, and combination strategies are now underway in the advanced/metastatic setting [39]. In these clinical trials, the endpoints used to assess the efficacy of Src inhibitors are focused solely on the primary tumor. Moreover, patients in these studies were typically followed for up to one year while receiving the inhibitor, with no long term follow up that would allow the potential impact of pan-SFK inhibitors on distant recurrences to be assessed, including the formation of liver metastases.

Pan-SFK inhibitors are known to target a broad spectrum of molecules. Dasatinib is an ATP competitive inhibitor of BCR-ABL, ephrin, c-KIT, PDGF receptor $\beta$, and targets all SFK members [40]. In our study, we have used concentrations below levels that result in offtarget effects, suggesting that Dasatinib is likely targeting SFKs in our system [41]. PP2 is considered to be a more specific inhibitor, and has different off target effects, when compared to Dasatinib [42]. Together, these considerations argue that members of the SFK are the bone fide targets in breast cancer cells and raise the intriguing possibility that specific members of the SFK could be engaged differentially to regulate Claudin- 2 expression, and the liver metastatic potential, in breast cancer cells.

Indeed, there are several SFK members, which include c-Src, Yes, Fyn, c-Fgr, Lyn, Lck, Hck, Blk, Yrk and Frk [43]. However, only a few of these SFK members are ubiquitous, including c-Src, Yes and Fyn; whereas others exhibit a more restricted pattern of expression in non-epithelial cells [43]. In breast cancer cells, loss of c-Src expression had no effect on Claudin-2 levels whereas diminished Yes or Fyn expression resulted in a dramatic upregulation of Claudin-2 levels. Thus, in the context of breast cancer cells, the dominant effect of PP2 or Dasatinib appears to be mediated through the inhibition of Yes or Fyn activity, which leads to higher Claudin-2 expression.

Through the use of Lyn-specific shRNAs and a Lynselective inhibitor, Bafetinib, we have identified Lyn as a potential therapeutic target to reduce liver metastatic ability of breast cancer cells. Our data comparing the effects of Dasatinib (a pan-SFK inhibitor) versus Bafetinib (a Lyn-selective inhibitor) suggest that specifically targeting Lyn would be recommended for the management of liver metastasis. Interestingly, Lyn has recently received attention as an important regulator of signaling in basal breast cancers, which represent the breast cancer models we have utilized in our current work, in which Lyn expression correlated with poor survival and increased likelihood of recurrence $[24,25]$. Numerous clinical trials have been completed to assess the effectiveness of Bafetinib in treating glioma, hormone refractory prostate cancer or B-CLL patients (NCT01234740, NCT01215799, 
NCT01144260, NCT00352677). To date, none of these clinical studies have examined the potential effect of Bafetinib treatment on the development of distant liver metastasis nor are we aware of any ongoing trials that plan to examine the efficacy of Bafetinib in the context of metastatic breast cancer. Our results support the consideration of Lyn-selective inhibitors, such as Bafetinib, in the treatment of breast cancer liver metastasis.

\section{MATERIALS AND METHODS}

\section{Cell culture}

The 4T1 and MDA-MB-231 cell lines were obtained from the American Type Culture Collection (ATCC). The generation of 4T1-derived liver-aggressive cell populations has been described previously [17]. BRC31 human breast cancer cells were kindly provided by Dr. Rancourt and cultured as previously described [26].

As previously reported, all lentiviral shRNA vectors were retrieved from the arrayed Mission ${ }^{\circledR}$ TRC genome-wide shRNA collections purchased from Sigma-Aldrich Corporation [44]. Additional information describing the shRNA vectors can be found at http://www.sigmaaldrich.com/life-science/functionalgenomics-and-rnai/shrna/library-information.html or http:// www.broad.mit.edu/genome_bio/trc/rnai.html, using the TRCN number. The following lentiviral shRNA vectors were used: shumanSRC, TRCN0000038150 and TRCN0000199313; shhuman $L Y N$, TRCN0000218210 and TRCN0000230901; shmouse $L Y N$, TRCN0000023664 and TRCN0000023665; shumanFYN, TRCN0000003097 and TRCN0000003101; shhumanYES1, TRCN0000010006 and TRCN0000121065. Lentiviral supernatants were generated as described at http:// www.broadinstitute.org/rnai/public/resources/protocols. Pooled stable populations were maintained under $1.5 \mu \mathrm{g} / \mathrm{ml}$ puromycin antibiotic selection.

\section{Explant cultures}

Explant cultures were derived from breast cancer patient-derived xenografts developed in-house (GCRC1735) or received as a kind gift from Alana Welm (HCI010) [30]. Briefly, excess breast tumor tissue from primary surgery was transported to the laboratory in icecold DMEM/F12, $50 \mu \mathrm{g} / \mathrm{ml}$ gentamicin, 1x penicillinstreptomycin, $2.5 \mu \mathrm{g} / \mathrm{ml}$ fungizone. Samples were cut into $1 \mathrm{~mm}^{3}$ fragments, covered in $50 \%$ matrigel, and transplanted into the mammary fat pad of 5-7 week-old NOG mice (Taconic, Hudson, NY, USA) under sterile conditions. When tumors reached $1 \mathrm{~cm}$ in the largest dimension, they were harvested aseptically, minced with sterile scalpels and dissociated at $37^{\circ} \mathrm{C}$ for $2-4$ hours on a rotisserie in digestion medium (RPMI, 2.5\% FBS, $10 \mathrm{mM}$ HEPES, $1 \mathrm{mg} / \mathrm{ml}$ collagenase type IV, $50 \mu \mathrm{g} / \mathrm{ml}$ gentamicin), 3-5 minutes in $0.25 \%$ trypsin/EDTA and then passed through a $40 \mu \mathrm{m}$ strainer. GCRC1735 breast tumor cells were cultured in adherent conditions, in which they were seeded on collagen I-coated plates in primary cell medium (DMEM, $5 \%$ FBS, $5 \mathrm{ng} / \mathrm{ml}$ human EGF, $5 \mu \mathrm{g} / \mathrm{ml}$ insulin, $1 \mu \mathrm{g} / \mathrm{ml}$ hydrocortisone, $35 \mu \mathrm{g} / \mathrm{ml}$ bovine pituitary extract, $50 \mu \mathrm{g} / \mathrm{ml}$ gentamicin). HCI010 breast tumor cells were cultured in non-adherent conditions, in which they were seeded on ultra-low attachment plates (Corning, Corning, NY, USA) in sphere medium (DMEM/F12, 1x B27, $20 \mathrm{ng} / \mathrm{ml}$ human EGF, $10 \mu \mathrm{g} / \mathrm{ml}$ insulin, $0.5 \mathrm{mg} /$ $\mathrm{ml}$ hydrocortisone, $20 \mathrm{ng} / \mathrm{ml} \mathrm{bFGF}, 10 \mu \mathrm{g} / \mathrm{ml}$ heparin, $50 \mu \mathrm{g} / \mathrm{ml}$ gentamicin). All human tissue was collected at McGill University Health Center in accordance with the protocols approved by the research ethics committee.

\section{Reagents}

Dasatinib (LC Laboratories, Woburn, MA, USA), PP2 (calbiochem, Gibbstown, NJ, USA) and Bafetinib (Selleckchem, Houston, TX, USA) were prepared at the appropriate concentrations using DiMethylSulfOxyde (DMSO). For all in vitro assays, cells were treated for 18 hours using the following concentrations: Dasatinib: $30 \mathrm{nM}$; PP2: $10 \mu \mathrm{M}$; Bafetinib: $10 \mu \mathrm{M}$.

\section{Immunoblotting}

Membranes were processed as previously described [17] and subjected to immunoblot analysis using the following antibodies: Claudin-2 (0.1 $\mu \mathrm{g} / \mathrm{ml}$; Cat. \#: 325600; Invitrogen, Burlington, ON, Canada), phospho-Src family kinase (Tyr 416) $(0.01 \mu \mathrm{g} / \mathrm{ml}$; Cat. \#: 2101), phospho-cFos (Ser 32) (0.035 $\mu \mathrm{g} / \mathrm{ml}$; Cat. \#: 5348), Lyn (0.015 $\mu \mathrm{g} /$ ml; Cat. \#: 2796), Fyn (0.07 $\mu \mathrm{g} / \mathrm{ml}$; Cat. \#: 4023), Yes (0.02 $\mu \mathrm{g} / \mathrm{ml}$; Cat. \#: 3201) (Cell Signaling, Whitby, ON, Canada), c-Src $(0.4 \mu \mathrm{g} / \mathrm{ml}$; Cat. \#: 05-184; Millipore, Billerica, MA, USA), c-Fos ( $0.4 \mu \mathrm{g} / \mathrm{ml}$; Cat. \#: sc-253X), phosphoc-Fos (Ser 374) $(0.2 \mu \mathrm{g} / \mathrm{ml}$; Cat. \#: sc-81485), c-Jun (0.4 $\mu \mathrm{g} / \mathrm{ml}$; Cat. \#: sc-1694X), phospho-c-Jun (Ser63) $(0.4 \mu \mathrm{g} / \mathrm{ml}$; Cat. \#: sc-822) (Santa Cruz Biotechnology, Dallas, TX, USA) and $\alpha$-tubulin $(0.5 \mu \mathrm{g} / \mathrm{ml}$; Cat. \#: T9026; Sigma, Oakville, ON, Canada). Blots were incubated with horseradish-peroxidase-conjugated anti-IgG secondary antibodies (Jackson ImmunoResearch Laboratories, Bar Harbor, ME, USA) and visualized with chemiluminescent HRP Substrate (Millipore, Billerica, MA, USA).

\section{Chromatin immunoprecipitation}

ChIP assays were conducted as described previously [45]. A detailed protocol could be found in supplementary methods.

\section{In vivo analysis following Dasatinib or Bafetinib treatment}

For experimental metastasis assays, parental 2776 liver-aggressive cells derived from the mouse 4T1 breast 
cancer cell line or 2776 cells harbouring shRNAs against

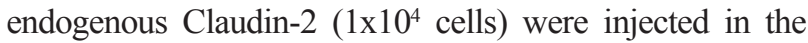
spleens of 6 to 8-week-old female Balb/c mice (Charles River, Senneville, QC, Canada) as previously described [17]. Dasatinib (10 mg/kg) was administered daily by oral gavage in $80 \mathrm{mmol} / \mathrm{L}$ citrate buffer, which was also used as vehicle control. Following splenic injection of the parental 2776 liver-aggressive cells ( $1 \times 10^{5}$ cells), Bafetinib (10 mg/kg) was administered daily by oral gavage in $0.5 \%$ methylcellulose, which was also used as vehicle control. Mice were sacrificed 12 days later and the number of lesion as well as the metastatic area/tissue area was quantified using Imagescope software (Aperio, Vista, CA, USA) as previously reported [17].

The mice were housed in facilities managed by the McGill University Animal Resources Centre and all animal experiments were conducted under a McGill University approved Animal Use Protocol in accordance with guidelines established by the Canadian Council on Animal Care.

\section{Statistical analysis}

Statistical significance values ( $P$ values) associated with liver metastasis formation from breast cancer cells injected in mice treated with Dasatinib (Figure 3) or Bafetinib (Figure 6) were calculated by performing a twosample unequal variance student's $t$-test.

\section{ACKNOWLEDGMENTS}

We acknowledge Ana Beatriz Toledo Dias (Henry C. Witelson Ocular Pathology Laboratory, Department of Ophtalmology, McGill University, Montreal, Canada) for Claudin-2 imuunohistochemical staining. We acknowledge the Goodman Cancer Research Centre histology core facility (McGill University) for routine histological services. We acknowledge Dr. Rancourt and Dr. Welm for providing us with cell lines and PDXs used in this study. We thank members of the Siegel laboratory and Dr. Josie Ursini-Siegel for thoughtful discussions and critical reading of the manuscript.

This work was supported by a program project grant from the Terry Fox Foundation (Grant \# 17003) and a CIHR operating grant (MOP 136907). B.E.H. and C.E.T. acknowledge support from the McGill Integrated Cancer Research Training Program, P.S. acknowledges support from a CIHR MD/Ph.D. studentship and from the CIHR/ McGill System Biology Training Program. Finally, P.M.S. acknowledges previous support from the Research Scholar (Junior II) program of the Fonds de recherche en santé du Québec (FRSQ) and is currently a McGill University William Dawson Scholar.

\section{CONFLICTS OF INTEREST}

The authors have no potential conflict of interest to disclose.

\section{REFERENCES}

1. Van den Eynden GG, Majeed AW, Illemann M, Vermeulen PB, Bird NC, Hoyer-Hansen G, Eefsen RL, Reynolds AR, Brodt P. The multifaceted role of the microenvironment in liver metastasis: biology and clinical implications. Cancer Res. 2013; 73:2031-2043.

2. Yardley DA. Visceral disease in patients with metastatic breast cancer: efficacy and safety of treatment with ixabepilone and other chemotherapeutic agents. Clinical breast cancer. 2010; 10:64-73.

3. Tabariès S, Siegel PM. Breast Cancer Liver Metastasis Springer. 2011; 16:273-303.

4. Runkle EA, Mu D. Tight junction proteins: from barrier to tumorigenesis. Cancer Lett. 2013; 337:41-48.

5. Martin T, Mason M, Jiang W. Tight junctions in cancer metastasis. Frontiers in bioscience: a journal and virtual library. 2011; 16:898.

6. Escudero-Esparza A, Jiang WG, Martin TA. The Claudin family and its role in cancer and metastasis. Front Biosci. 2011; 16:1069-1083.

7. Kinugasa T, Huo Q, Higashi D, Shibaguchi H, Kuroki M, Tanaka T, Futami K, Yamashita Y, Hachimine K, Maekawa S, Nabeshima K, Iwasaki H. Selective upregulation of claudin-1 and claudin-2 in colorectal cancer. Anticancer Res. 2007; 27:3729-3734.

8. Weber CR, Nalle SC, Tretiakova M, Rubin DT, Turner JR. Claudin-1 and claudin-2 expression is elevated in inflammatory bowel disease and may contribute to early neoplastic transformation. Lab Invest. 2008; 88:1110-1120.

9. Buchert M, Papin M, Bonnans C, Darido C, Raye WS, Garambois V, Pelegrin A, Bourgaux JF, Pannequin J, Joubert D, Holle F. Symplekin promotes tumorigenicity by up-regulating claudin-2 expression. Proc Natl Acad Sci U S A. 2010; 107:2628-2633.

10. Dhawan P, Ahmad R, Chaturvedi R, Smith J, Midha R, Mittal M, Krishnan M, Chen X, Eschrich S, Yeatman T. Claudin-2 expression increases tumorigenicity of colon cancer cells: role of epidermal growth factor receptor activation. Oncogene. 2011; 30:3234-3247.

11. Patonai A, Erdelyi-Belle B, Korompay A, Somoracz A, Straub BK, Schirmacher P, Kovalszky I, Lotz G, Kiss A, Schaff Z. Claudins and tricellulin in fibrolamellar hepatocellular carcinoma. Virchows Archiv : an international journal of pathology. 2011; 458:679-688.

12. Jung H, Jun KH, Jung JH, Chin HM, Park WB. The expression of claudin-1, claudin-2, claudin-3, and claudin-4 in gastric cancer tissue. The Journal of surgical research. 2011; 167:e185-191.

13. Holczbauer A, Gyongyosi B, Lotz G, Szijarto A, Kupcsulik P, Schaff Z, Kiss A. Distinct claudin expression profiles of hepatocellular carcinoma and metastatic colorectal and pancreatic carcinomas. The journal of histochemistry 
and cytochemistry: official journal of the Histochemistry Society. 2013; 61:294-305.

14. Kim T, Huh J, Lee S, Kang H, Kim G, An H. Downregulation of claudin-2 in breast carcinomas is associated with advanced disease. Histopathology. 2008; 53:48-55.

15. Szasz A, Tokes A, Micsinai M, Krenacs T, Jakab C, Lukacs L, Nemeth Z, Baranyai Z, Dede K, Madaras L. Prognostic significance of claudin expression changes in breast cancer with regional lymph node metastasis. Clinical and Experimental Metastasis. 2011; 28:55-63.

16. Tabariès S, Dupuy F, Dong Z, Monast A, Annis MG, Spicer J, Ferri LE, Omeroglu A, Basik M, Amir E, Clemons M, Siegel PM. Claudin-2 promotes breast cancer liver metastasis by facilitating tumor cell interactions with hepatocytes. Mol Cell Biol. 2012; 32:2979-2991.

17. Tabariès S, Dong Z, Annis MG, Omeroglu A, Pepin F, Ouellet V, Russo C, Hassanain M, Metrakos P, Diaz Z, Basik M, Bertos N, Park M, Guettier C, Adam R, Hallett M, et al. Claudin-2 is selectively enriched in and promotes the formation of breast cancer liver metastases through engagement of integrin complexes. Oncogene. 2011; 30:1318-1328.

18. Kimbung S, Kovacs A, Bendahl PO, Malmstrom P, Ferno M, Hatschek T, Hedenfalk I. Claudin-2 is an independent negative prognostic factor in breast cancer and specifically predicts early liver recurrences. Molecular oncology. 2014; 8:119-128.

19. Dhawan P, Ahmad R, Chaturvedi R, Smith JJ, Midha R, Mittal MK, Krishnan M, Chen X, Eschrich S, Yeatman TJ, Harris RC, Washington MK, Wilson KT, Beauchamp RD, Singh AB. Claudin-2 expression increases tumorigenicity of colon cancer cells: role of epidermal growth factor receptor activation. Oncogene. 2011; 30:3234-3247.

20. Bromann PA, Korkaya H, Courtneidge SA. The interplay between Src family kinases and receptor tyrosine kinases. Oncogene. 2004; 23:7957-7968.

21. Ishizawar R, Parsons SJ. c-Src and cooperating partners in human cancer. Cancer Cell. 2004; 6:209-214.

22. Finn RS. Targeting Src in breast cancer. Ann Oncol. 2008; 19:1379-1386.

23. Summy JM, Gallick GE. Treatment for advanced tumors: SRC reclaims center stage. Clin Cancer Res. 2006; 12:1398-1401.

24. Choi YL, Bocanegra M, Kwon MJ, Shin YK, Nam SJ, Yang JH, Kao J, Godwin AK, Pollack JR. LYN is a mediator of epithelial-mesenchymal transition and a target of dasatinib in breast cancer. Cancer Res. 2010; 70:2296-2306.

25. Hochgrafe F, Zhang L, O'Toole SA, Browne BC, Pinese M, Porta Cubas A, Lehrbach GM, Croucher DR, Rickwood D, Boulghourjian A, Shearer R, Nair R, Swarbrick A, Faratian D, Mullen P, Harrison DJ, et al. Tyrosine phosphorylation profiling reveals the signaling network characteristics of Basal breast cancer cells. Cancer Res. 2010; 70:9391-9401.
26. Laplante Y, Rancourt C, Poirier D. Relative involvement of three 17beta-hydroxysteroid dehydrogenases (types 1, 7 and 12) in the formation of estradiol in various breast cancer cell lines using selective inhibitors. Molecular and cellular endocrinology. 2009; 301:146-153.

27. Neve RM, Chin K, Fridlyand J, Yeh J, Baehner FL, Fevr T, Clark L, Bayani N, Coppe JP, Tong F, Speed T, Spellman PT, DeVries S, Lapuk A, Wang NJ, Kuo WL, et al. A collection of breast cancer cell lines for the study of functionally distinct cancer subtypes. Cancer Cell. 2006; 10:515-527.

28. Ikari A, Sato T, Watanabe R, Yamazaki Y, Sugatani J. Increase in claudin-2 expression by an EGFR/MEK/ERK/cFos pathway in lung adenocarcinoma A549 cells. Biochim Biophys Acta. 2012; 1823:1110-1118.

29. Monje P, Marinissen MJ, Gutkind JS. Phosphorylation of the carboxyl-terminal transactivation domain of c-Fos by extracellular signal-regulated kinase mediates the transcriptional activation of AP-1 and cellular transformation induced by platelet-derived growth factor. Mol Cell Biol. 2003; 23:7030-7043.

30. DeRose YS, Wang G, Lin YC, Bernard PS, Buys SS, Ebbert MT, Factor R, Matsen C, Milash BA, Nelson E, Neumayer L, Randall RL, Stijleman IJ, Welm BE, Welm AL. Tumor grafts derived from women with breast cancer authentically reflect tumor pathology, growth, metastasis and disease outcomes. Nat Med. 2011; 17:1514-1520.

31. Kantarjian H, le Coutre P, Cortes J, Pinilla-Ibarz J, Nagler A, Hochhaus A, Kimura S, Ottmann O. Phase 1 study of INNO-406, a dual Abl/Lyn kinase inhibitor, in Philadelphia chromosome-positive leukemias after imatinib resistance or intolerance. Cancer. 2010; 116:2665-2672.

32. Santos FP, Kantarjian H, Cortes J, Quintas-Cardama A. Bafetinib, a dual Bcr-Abl/Lyn tyrosine kinase inhibitor for the potential treatment of leukemia. Current opinion in investigational drugs. 2010; 11:1450-1465.

33. Yokota A, Kimura S, Masuda S, Ashihara E, Kuroda J, Sato K, Kamitsuji Y, Kawata E, Deguchi Y, Urasaki Y, Terui $\mathrm{Y}$, Ruthardt $\mathrm{M}$, Ueda $\mathrm{T}$, Hatake $\mathrm{K}$, Inui $\mathrm{K}$, Maekawa T. INNO-406, a novel BCR-ABL/Lyn dual tyrosine kinase inhibitor, suppresses the growth of $\mathrm{Ph}+$ leukemia cells in the central nervous system, and cyclosporine A augments its in vivo activity. Blood. 2007; 109:306-314.

34. Deguchi Y, Kimura S, Ashihara E, Niwa T, Hodohara K, Fujiyama Y, Maekawa T. Comparison of imatinib, dasatinib, nilotinib and INNO-406 in imatinib-resistant cell lines. Leukemia research. 2008; 32:980-983.

35. Zhang Y, Pu X, Shi M, Chen L, Qian L, Song Y, Yuan G, Zhang H, Yu M, Hu M, Shen B, Guo N. c-Jun, a crucial molecule in metastasis of breast cancer and potential target for biotherapy. Oncol Rep. 2007; 18:1207-1212.

36. Gucalp A, Sparano JA, Caravelli J, Santamauro J, Patil S, Abbruzzi A, Pellegrino C, Bromberg J, Dang C, Theodoulou M, Massague J, Norton L, Hudis C, Traina TA. 
Phase II trial of saracatinib (AZD0530), an oral SRCinhibitor for the treatment of patients with hormone receptor-negative metastatic breast cancer. Clinical breast cancer. 2011; 11:306-311.

37. Finn RS, Bengala C, Ibrahim N, Roche H, Sparano J, Strauss LC, Fairchild J, Sy O, Goldstein LJ. Dasatinib as a single agent in triple-negative breast cancer: results of an open-label phase 2 study. Clin Cancer Res. 2011; 17:6905-6913.

38. Mayer EL, Baurain JF, Sparano J, Strauss L, Campone M, Fumoleau P, Rugo H, Awada A, Sy O, Llombart-Cussac A. A phase 2 trial of dasatinib in patients with advanced HER2-positive and/or hormone receptor-positive breast cancer. Clin Cancer Res. 2011; 17:6897-6904.

39. Elsberger B. Translational evidence on the role of Src kinase and activated Src kinase in invasive breast cancer. Critical reviews in oncology/hematology. 2014; 89:343-351.

40. Li J, Rix U, Fang B, Bai Y, Edwards A, Colinge J, Bennett KL, Gao J, Song L, Eschrich S, Superti-Furga G, Koomen J, Haura EB. A chemical and phosphoproteomic characterization of dasatinib action in lung cancer. Nature chemical biology. 2010; 6:291-299.
41. Karaman MW, Herrgard S, Treiber DK, Gallant P, Atteridge CE, Campbell BT, Chan KW, Ciceri P, Davis MI, Edeen PT, Faraoni R, Floyd M, Hunt JP, Lockhart DJ, Milanov $\mathrm{ZV}$, Morrison MJ, et al. A quantitative analysis of kinase inhibitor selectivity. Nature biotechnology. 2008; 26:127-132.

42. Bain J, Plater L, Elliott M, Shpiro N, Hastie CJ, McLauchlan H, Klevernic I, Arthur JS, Alessi DR, Cohen P. The selectivity of protein kinase inhibitors: a further update. The Biochemical journal. 2007; 408:297-315.

43. Gelman IH. Src-family tyrosine kinases as therapeutic targets in advanced cancer. Front Biosci (Elite Ed). 2011; 3:801-807.

44. Huang S, Holzel M, Knijnenburg T, Schlicker A, Roepman P, McDermott U, Garnett M, Grernrum W, Sun C, Prahallad A, Groenendijk FH, Mittempergher L, Nijkamp W, Neefjes J, Salazar R, Ten Dijke P, et al. MED12 controls the response to multiple cancer drugs through regulation of TGF-beta receptor signaling. Cell. 2012; 151:937-950.

45. Deblois G, Chahrour G, Perry MC, Sylvain-Drolet G, Muller WJ, Giguere V. Transcriptional control of the ERBB2 amplicon by ERRalpha and PGC-1beta promotes mammary gland tumorigenesis. Cancer Res. 2010; 70:10277-10287. 